\title{
Research of Zero-crossing detection for Sensorless BLDC motor
}

\author{
Ling yun \\ College of Computer Science and Technology \\ Soochow University \\ SuZhou,China \\ lingyun2012@suda.edu.cn
}

\author{
ZHU le-le \\ Soochow University \\ SuZhou,China \\ College of Computer Science and Technology \\ zhulele_12@126.com
}

\author{
WANG yi-huai \\ College of Computer Science and Technology \\ Soochow University \\ SuZhou,China \\ wangyihuai@suda.edu.cn
}

\begin{abstract}
The key technology of Sensorless BLDC motor control is Zero-crossing detection. Based on full research and analysis of the Back-EMF motor principle, wave and traditional detection methods, a new method of Back-EMF Zero-crossing detection is introduced. The method does not rigidly adhere to the principles of traditional back-EMF zero-crossing detection, rejected the midpoint motor, depth filter and voltage comparison circuits in the design of the traditional zero-crossing detection circuit, gets commutation points by realizing three-phase voltage real time collection, filtering and designing the effective software algorithms. The method is applied in the electric car control system. The experiment result shows that the method has some advantages which are simple zero-crossing detection circuits, software with clear structure and good real-time and so on.
\end{abstract}

Key words: Back-EMF Zero-crossing detection; BLDC motor; Sensorless; DSC(Digital Signal Controller); software

\section{INTRODUCTION}

Sensorless BLDC motor is a kind of BLDC, not only has many of the advantages of BLDC, but also solved many problems of Sensorless BLDC, so it has been widely used in various industries [1][2]. The key technology of Sensorless BLDC motor control is Zero-crossing detection, and it has been a research focus at home and abroad. There have been many detection methods for different applications [3]. These methods calculate position of the rotor by collecting motor operating parameters, such as current, voltage, back-EMF. There are several common zero-crossing detection, such as Freewheeling diode method, the current detection method and back-EMF method. Freewheeling diode method is essentially a kind of back-EMF method, applied to control low-speed motor. But because the need to use the six isolated power supply, it led to the hardware costs and system instability. In order to determine the position of the rotor, the current detection method, as known as the inductance detection method, use the correspondence between the internal inductance and rotor position of the motor windings to detect the inductor. Because the method requires real-time acquisition winding inductance, it is rarely used in practical. Back-EMF method is a best one, because it is the most technologically matured, the most effective and most widely used. [4]。 The method is based on ignore the armature reaction, get the six key position of running motor by detecting the conduction phase back-EMF zero crossing and delaying of $30^{\circ}$. The traditional back-EMF method is to use the hardware circuit detection method, it uses DC bus voltage midpoint to reconstruct the motor midpoint and requires a hardware filter and the voltage comparator circuit, so it increases the hardware cost and system instability. [5] In addition, because the factors of switching noise, the depth filter circuit, voltage comparator circuit, the method easily lead to back-EMF error zero crossing signal and commutation delay. [6]。

In order to solve the existing problems of the Back-EMF, the paper propose a new method, Back-EMF Zero Crossing Detection Method based on Embedded Software, called as ES-BEZ. The method rejects the midpoint motor, depth filter and voltage comparison circuits in the design of the traditional zero-crossing detection circuit, gets commutation points by realizing three-phase voltage real time collection, filtering and designing the effective software algorithms. In addition, the rapid development of embedded technology also contributes to the rapid innovation of traditional technologies. Traditional methods cannot meet the requirements of the current technology, which requires new theories and methods to adapt to new technology, so, ES-BEZ of the proposed for BLDC field has an important reference.

\section{ES-BEZ THEORETICAL BASIS AND ALGORITHM}

\section{ANALYSIS}

BLDC motor is commonly used three-phase six state conduction, and it needs six rotor positions for the motor commutation in one cycle. Fig. 1 shows the equivalent circuit and driver circuit diagram of the BLDC motor. Ud is power, V1 V6 are FET, and $\mathrm{n}$ is motor winding midpoint. Assumptions ignore the interference of the eddy current, hysteresis, and sawtooth effect, Motor three-phase 
terminal voltage equations have been proposed form Fig. $1[7]$.

$$
\left[\begin{array}{l}
U a \\
U b \\
U c
\end{array}\right]=\left[\begin{array}{ccc}
R & 0 & 0 \\
0 & R & 0 \\
0 & 0 & R
\end{array}\right]\left[\begin{array}{c}
i_{a} \\
i_{b} \\
i_{c}
\end{array}\right]+p\left[\begin{array}{ccc}
L & 0 & 0 \\
0 & L & 0 \\
0 & 0 & L
\end{array}\right]\left[\begin{array}{c}
i_{a} \\
i_{b} \\
i_{c}
\end{array}\right]+\left[\begin{array}{c}
e_{a} \\
e_{b} \\
e_{c}
\end{array}\right]+\left[\begin{array}{c}
U n \\
U n \\
U n
\end{array}\right]
$$

$\mathrm{Ua}, \mathrm{Ub}, \mathrm{Uc}$ are the three-phase motor terminal voltage, Un is the motor midpoint voltage, ia, ib, ic are three-phase motor winding current, ea, eb, ec are the three-phase back-EMF, $\mathrm{R}$ is the stator resistance, Ls is the stator winding self-inductance, $\mathrm{Lm}$ is the stator winding mutual inductance, $\mathrm{L}=\mathrm{Ls}-\mathrm{Lm}$ is effective inductance, $\mathrm{p}$ is the differential operator.

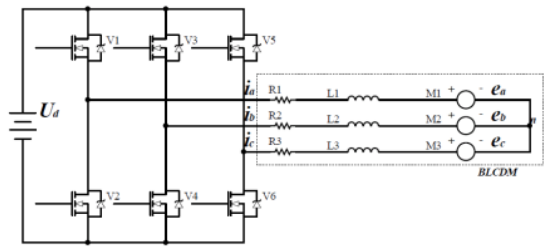

Figure 1. The equivalent circuit and driver circuit diagram of the BLDC motor

The following equations can be got by subtracting each other among $\mathrm{Ua} 、 \mathrm{Ub} 、 \mathrm{Uc}$.

$$
\left\{\begin{array}{l}
U a b=R\left(i_{a}-i_{b}\right)+p L\left(i_{a}-i_{b}\right)+\left(e_{a}-e_{b}\right) \\
U a c=R\left(i_{a}-i_{c}\right)+p L\left(i_{a}-i_{c}\right)+\left(e_{a}-e_{c}\right) \\
U b c=R\left(i_{b}-i_{c}\right)+p L\left(i_{b}-i_{c}\right)+\left(e_{b}-e_{c}\right)
\end{array}\right.
$$

Due to only two-phase conduction when a motor running, we assume that $\mathrm{a}$ and $\mathrm{b}$ phase conduction, $\mathrm{c}$ phase cut-off, simplified equation (2) have to formula (3).

$$
U a c-U c b=R\left(i_{a}+i_{b}-2 i_{c}\right)+p L\left(i_{a}+i_{b}-2 i_{c}\right)+\left(e_{a}+e_{b}-2 e_{c}\right)
$$

Voltage commutation principle diagram of Sensorless BLDC motor is showed in Fig. 2. Z1 Z6 are zero points, S1 S6 are voltage commutation points, V1 V6 are on-off state. Through the analysis Fig. 2, we can draw the conclusion that the actual commutation and zero point difference between $30^{\circ}$.

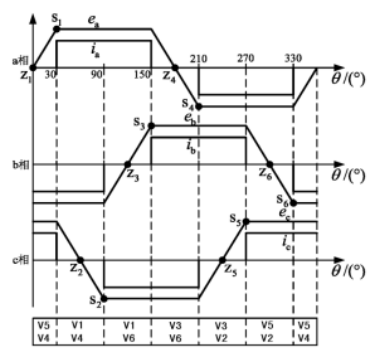

Figure 2. Voltage commutation principle diagram of Sensorless BLDC motor

In the near of c phase Zero-crossing point, $e_{a}+e_{b}=0$. In addition, according to the motor winding star connection, we can know $i_{a}+i_{b}+i_{c}=0$. Formula (3) can be simplified for the following formula (4).

$$
e_{c}=0.5\left((U c b-U a c)-3 R i_{c}-3 p L i_{c}\right)
$$

The back-EMF of $\mathrm{c}$ phase has relationship with the following physical quantities, voltage of $\mathrm{cb}$ 、 ac, current of $c$ phase, motor internal resistance and inductance. We think that cut-off phase current is 0 after a period of motor commutation. So $i_{c}=0$, formula (4) can be further simplified.

$$
e_{c}=0.5(U c b-U a c)
$$

The back-EMF of c phase has relationship with the a, $\mathrm{b}, \mathrm{c}$ phases voltage. When rotor infinite close to zero point, ec is 0. Formula (5) can be simplified as $2 \mathrm{Uc}=\mathrm{Ua}+\mathrm{Ub}$. In other words, as long as the real-time acquisition machine three terminal voltage, through the formula (5) real-time calculation we can detect the zero-crossing point.

\begin{tabular}{|c|c|c|}
\hline intervalV1V2V3V4V5V6 & conditions & Exchange \\
\hline on off off on off off & $e_{c} \leq 0$ & $a, b->a, c$ \\
\hline on off off off off on & $e_{c} \geq 0$ & $a, c->b, c$ \\
\hline off off on off off on & $e_{c} \leq 0$ & $b, c->b, a$ \\
\hline 4 off on on off off off & $e_{c} \geq 0$ & $b, a->c, a$ \\
\hline off on off off on off & $e_{c} \leq 0$ & $c, a->c, b$ \\
\hline off off off on on off & $e_{c} \geq 0$ & $c, b->a, b$ \\
\hline
\end{tabular}

Table 1 is relationship of Back-EMF and conducting interval.

Table 1 Counter electromotive force and conducting interval relationship

From the circuit Angle consideration, ES-BEZ only need resistance partial pressure circuit, but the traditional method need hardware construct resistance network neutral or bus voltage midpoint, through the voltage comparison circuit to realize counter electromotive force the zero examination. From algorithm, ES-BEZ through the acquisition motor three-phase terminal voltage, and formula (5) calculations counter electromotive force zero crossing detection, but traditional method mainly through the capture compare the output level signal. ES - BEZ, compared with the traditional method, has many advantages, such as the circuit simple, hardware dependence is weak, anti-jamming strong, not easy to receive external signal interference, as so on. So, ES-BEZ is a new method, that better traditional method, it is put forward for brushless dc motor area has important reference meaning.

\section{ES-BEZ SOFTWARE ALGORITHM}

The paper chooses MC56F8257 [8] which is one of series microcontroller Freescale DSC for master control chip, and design the ES-BEZ software algorithm. A set of perfect motor master controller software including the following several parts, motor start algorithm, counter electromotive force zero crossing detection, motor commutation control and speed control. It uses PWM (Pulse Width Modulation) to adjust the speed [9]. According to the ES-BEZ algorithm thought combining MC56F8257 characteristic, the article design ES - BEZ software algorithm. The program design, it joined the components program design thought. This kind of design thought to help strengthen program algorithm structure of the resolution. 


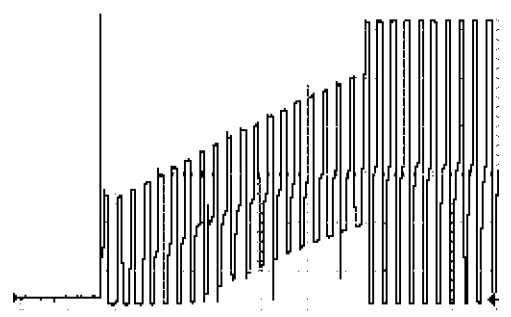

Figure 3. When the PWM duty ratio $40 \%$, c phase voltage waveform diagram

A and b phase conduction, c phase cut-off in the Fig. 3, corresponding to the interval 4 of the table 1 . Observation in Fig. 3, you can see much clutter. Therefore, we need to consider how to remove filter voltage, get the correct data.

According to the master control chip MC56F8257 PWM module performance, it is known that in the PWM each cycle a timer interrupt overflow can triggered. So when PWM mode is align center, the voltage for effective value. Based on the above ideas, this section realized ES-BEZ software algorithm. ES-BEZ software algorithm flow chart shows in Fig. 4.

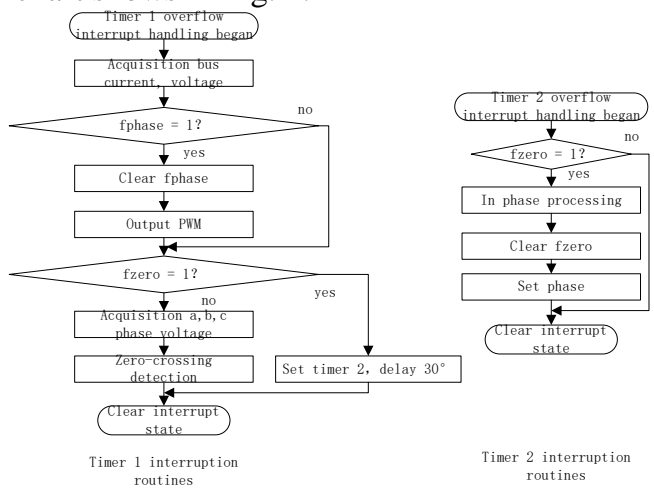

Figure 4. ES-BEZ software algorithm flow chart

Timer 1 interrupts are decided by the PWM cycle, in the timer 1 overflow interrupt routine, algorithm collected bus voltage, bus current and voltage of the three-phase motor, and according to the data collected by formula (5) and table 1 determine whether detection to zero signal. Setting timer 2 after zero signal, it delay $30^{\circ}$ for commutation.

Through the software algorithm performance analysis, interrupt mode to realize the zero examination phase delay of small, high real-time performance, assuming PWM frequency for $16 \mathrm{KHZ}$, can be calculated per second the zero frequency of testing for 8000 times.

\section{ES-BEZ ALGORITHM TEST ANALYSIS}

In order to validate ES-BEZ performance, this paper use ES-BEZ software algorithm electric car counter electromotive force the zero examination. ES-BEZ software algorithm using the main hardware circuit is electric pressure circuit of subsection [10], this circuit, and the specific design as shown in Fig. 5.

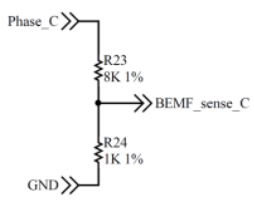

Figure 5. Single-phase voltage acquisition circuit

Phase_C is c phase voltage, BEMF_sense_C is signal acquisition point, GND is ground. The relationship of acquisition voltage with actual voltage is VBEMF_sense_C $=1 / 9 *$ VPhase_C。

In experimental testing, we observed motor have abnormal vibration and noise. The result of preliminary analysis is that the motor phase lack. After many experiments, we found that the motor commutation time is not accurate. Repeated analysis ES-BEZ, we found that formula (5) overlooked current of phase commutation. According to the characteristics of inductance, commutation time winding fly-wheel, produce the degaussing event. Fig. 6 for motor $\mathrm{c}$ phase terminal voltage waveform diagram. Comparing with Fig. 2, we can find that the circle area is degaussing event of $\mathrm{c}$ phase commutation.

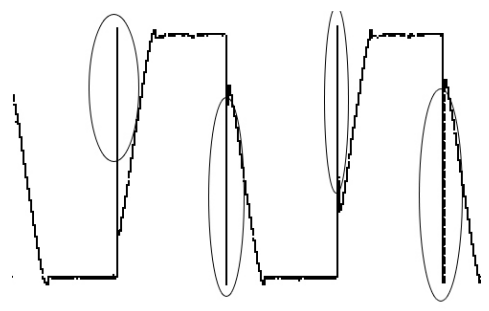

Figure 6. C phase voltage waveform diagram

In order to avoid mistake zero judgment, ES-BEZ provisions in phase from the fourth timer 1 overflow interrupt began to zero crossing detection. At the same time, ES-BEZ across from zero detection made certain conditions, only when the collection phase voltage in a bus voltage between $20 \% \sim 80 \%$ to execute counter electromotive force zero crossing detection algorithm. In the correction of the above problems, the experimental observation found that motor sometimes will still appear afore-mentioned phenomenon, analysis found that each PWM cycle can produce two timer 1 overflow interrupt, PWM end of overflow interrupt, can cause program error handling. Based on the modified ES-BEZ again, when conducting phase forward voltage is less than $90 \%$ of the bus voltage, the overflow interrupt is not done zero detection processing.

\section{CONCLUDING REMARKS}

This paper make full use of embedded software algorithm for sensorless brushless dc motor of the zero crossing detection, and put forward the zero crossing detection ES-BEZ method, has the hardware circuit concise, software structure is clear and good real-time etc. Its core idea is deduced $e_{c}=0.5(U c b-U a c)$, and calculates the counter electromotive force a zero, combining hardware interrupt, again late $30^{\circ}$ Angle in phase. Concurrent programming method makes the 
software algorithm has high efficiency, components programming thought makes the software algorithm has a clear structure. The practical application in electric vehicle control system, the result shows that this method hardware dependence is weak, anti-jamming strong guarantee the system's real time, it is a kind of sensorless brushless dc motor control with the new hardware and software design methods.

\section{REFERENCES}

[1] Kenichi I, Hideo U, Minoru K, etc. Microcomputer control for sensorless brushless motor [J]. IEEE Transactions on Industry Applications, 1985,21(4):595-601.

[2] Su G J, Mckeever J W. Low-cost sensorless control of brushless dc motors with improved speed range[J]. IEEE Transactions on Power Electronics, 2004, 19(2):296-302.

[3] Shen J X, Iwasaki S. Sensorless control of ultrahigh-speed PM brushless motor using PLL and third harmonic back EMF[J]. IEEE Transactions on Industrial Electronics, 2006,53(2):421-428.
[4] Lin C T, Hung C W, Liu C W. Position sensorless control for four-switch three-phase brushless DC motor drives[J]. IEEE Transactions on Power Electronics, 2008, 23(1):438-444.

[5] Chen C H, Cheng M Y. A new cost effective sensorless commutation method for brushless DC motors without phase shift circuit and neutral voltage[J]. IEEE Transactions on Power Electronics, 2007, 22(2):644-653.

[6] Ni J, Wu L J, Zhang B, etc. A novel adaptive commutation angle method for single phase BLDC motor. in:Proceedings of International Conference on Electrical Machines and Systems[J]. Seoul, 2007, 446-449.

[7] Wu H X, Cheng S K, Cui S M. A controller of brushless de motor for electric vehicle[J]. IEEE Transactions on Magnetics, 2005,41(1):509-513.

[8] Freescale Semiconductor, Inc. MC56F825XRM Rev.2[EB/OL]. (2010-10). http://cache. freescale. com/files/dsp/doc/ref_manual/MC56F825XRM.pdf?fpsp=1.

[9] Lai Y S, Lin Y K. Assessment pulse-width modulation techniques for brushless dc motor drives[J]. IEEE Industry Applications Magazine, 2008, 14(5):34-44.

[10] XIONG Hui, LI Yukun, You Yiming . A Back-EMF control method of BLDCM based on S12X MCU[J]. Application of Electronic Technique, 2010,2:95- 97. 